\title{
Comparison of Troponin I levels versus myocardial dysfunction on prognosis in sepsis
}

\author{
Francesca Innocenti ${ }^{1}\left[\right.$ - Vittorio Palmieri ${ }^{2}$. Valerio Teodoro Stefanone ${ }^{1} \cdot$ Federico D'Argenzio $^{1} \cdot$ Marco Cigana $^{1}$. \\ Michele Montuori ${ }^{1}$ - Elisa Capretti ${ }^{1} \cdot$ Anna De Paris $^{1} \cdot$ Stefano Calcagno $^{1} \cdot$ Irene Tassinari $^{1} \cdot$ Riccardo Pini $^{1}$
}

Received: 10 November 2020 / Accepted: 8 March 2021

(c) Società Italiana di Medicina Interna (SIMI) 2021

\begin{abstract}
In the context of sepsis, we tested the relationship between echocardiographic findings and Troponin, and their impact on prognosis. In this prospective study, we enrolled 325 septic patients (41\% with shock), not mechanically ventilated, between October, 2012 and June, 2019 among those admitted to our High-Dependency Unit. By echocardiography within $24 \mathrm{~h}$ from the admission, sepsis-induced myocardial dysfunction (SIMD) was defined as left ventricular (LV) systolic dysfunction (speckle-tracking-based global longitudinal peak systolic strain, GLS, > -14\%) and/or right ventricular (RV) systolic dysfunction (Tricuspid Annular Plane Systolic Excursion, TAPSE $<16 \mathrm{~mm}$ ). Troponin I levels were measured upon admission (T0) and after $24 \mathrm{~h}$ (T1); it was considered normal if $>0.1 \mathrm{ng} / \mathrm{mL}$. Mortality was assessed at day-7 and day-28 end-points. One-hundred and forty-two patients had normal Troponin level at T0 and T1 (G1), 69 had abnormal levels at T0 or T1 (G2) and 114 showed abnormal Troponin levels at both T0 and T1 (G3). Compared to G1, patients in G3 had worse LV and RV systolic function (GLS $-11.6 \pm 3.4 \%$ vs $-14.0 \pm 3.5 \%, p<0.001$; TAPSE $18 \pm 0.5$ vs $19 \pm 0.5 \mathrm{~mm}, p=0.047$ ) and greater day-28 (34\% vs $20 \%, p=0.015)$ mortality. In a Cox survival analysis including age, Troponin and SOFA score, mortality was predicted by the presence of SIMD (RR 3.24, 95\% CI 1.72-6.11, p <0.001) with no contribution of abnormal Troponin level. While abnormal Troponin levels were associated with SIMD diagnosed by echocardiography, only the presence of SIMD predicted the short- and medium-term mortality rate, without an independent contribution of increased Troponin levels.
\end{abstract}

Keywords Sepsis · Troponin · Sepsis-associated myocardial dysfunction $\cdot$ Prognosis

\section{Introduction}

Cardiac Troponins are well-known markers of myocardial injury. Up to $60 \%$ of patients admitted to the Intensive Care Units (ICU) show an elevation in their level from non-coronary causes [1]. In the presence of sepsis, the proportion of patients with a raised cardiac Troponin reaches the $80 \%$ [2] and its prognostic performance has already been investigated in numerous studies. Several authors demonstrated an association between elevated Troponin and an increased

Francesca Innocenti innocenti.fra66@gmail.com

1 High-Dependency Unit, Department of Clinical and Experimental Medicine, Azienda OspedalieroUniversitaria Careggi, Lg. Brambilla 3, 50134 Firenze, Italy

2 Transplant Cardiosurgery Unit, Department of Cardiac Surgery and Transplant, Ospedale Dei Colli Monaldi-Cotugno-CTO, Naples, Italy mortality rate [3-8], but the prognostic role of this biomarker remains a topic of debate [9-11].

Beyond its prognostic role, the accuracy of Troponin for the diagnosis of sepsis-induced myocardial dysfunction (SIMD) has been tested [12-14]. In septic patients, a newonset cardiac dysfunction occurs in about $60 \%$ of patients and may involve the left ventricle (LV), the right ventricle (RV) or both. The diagnosis of SIMD actually relies only on the echocardiographic evaluation, and the relationship between the presence of echocardiographically detectable myocardial systolic or diastolic dysfunction and the level of Troponin is actually a topic of debate. Several authors demonstrated the correlation between an increased level of cardiac biomarkers and an abnormal LV ejection fraction (EF) and indexes of diastolic dysfunction [15, 16]. De Geer et al. demonstrated a correlation between abnormal Global Longitudinal Strain (GLS), a measure of LV systolic function less load dependent, compared to conventional parameters, and an increased level of NTproBNP and Troponin 
[17]. However, a defined cutoff level of Troponin on which to base the diagnosis of SIMD has never been defined.

The aims of the present study were: (1) to test the diagnostic accuracy of elevated Troponin I for the presence of SIMD, with identification of LV and RV systolic dysfunction respectively by GLS and Tricuspidal Annular Plane Systolic Excursion (TAPSE); and (2) to evaluate the prognostic value of Troponin I for the day-7 and day-28 mortality rates.

\section{Methods}

\section{Study design and setting}

The "Toscana - Area vasta-Centro" inter-institutional ethic committee approved the study (registration number OSS.13.031), which was conducted in accordance with the Helsinki Declaration of 1964 (revised 2008). All patients gave informed consent to enter the study.

In this observational prospective study, we included consecutive patients admitted to the Emergency DepartmentHigh-Dependency Unit (ED-HDU) between October 2012 and June 2019. Consecutive patients with diagnosis of sepsis were evaluated longitudinally to assess impact of Troponin vs echocardiographically defined myocardial systolic dysfunction on very short and short-term all-cause mortality. Sepsis diagnosis was based on 2001 criteria [18] for patients included until June 2016; since then, patients were selected based on Sepsis-3 criteria [19, 20].

We excluded patients in whom LV walls could not be evaluated through standard apical views, as well as those with left heart valvular disease more than moderate. Patients with chest pain and/or diagnostic ECG abnormalities for acute myocardial ischemia were excluded. No patient was on mechanical ventilation as ED-HDU is a sub-intensive care unit with advanced monitoring and treatments, excluding patients who needed invasive ventilators.

Criteria to define presence of coronary artery disease (CAD), chronic kidney disease (CKD), chronic obstructive pulmonary disease (COPD) and neoplasia have already been defined elsewhere [21]. A pre-existing LV or RV systolic dysfunction was defined in the presence of a previous imaging test in medical records. We collected clinical and laboratory data upon the admission to the ED (T0) and re-evaluated within $24 \mathrm{~h}$ from the admission (T1); at the same time intervals, the Sequential Organ Failure Assessment (SOFA) score was calculated. SOFA score was dichotomized as $<$ or $\geq 5$, based on a previous report where we showed that a SOFA score $>5$ was associated with a unfavorable prognosis in septic patients admitted to the EDHDU [22]. Age was dichotomized as $<$ or $\geq 65$ years, based on the standard cutoff to define elderly people. Troponin I was measured upon ED admission and after $24 \mathrm{~h}$ and it was considered normal if lower than $0.1 \mathrm{ng} / \mathrm{mL}$, which is the conventional normal upper limit. Both T0 and T1 values were dichotomized based on the normal value. Thereafter, the study population was divided in three subgroups: (1) G1, patients with both $\mathrm{T} 0$ and $\mathrm{T} 1$ value in the normal range $(<0.1 \mathrm{ng} / \mathrm{mL}) ; 2) \mathrm{G} 2$, patients with either T0 or T1 value in the abnormal range; (3) G3, patients with both values in the abnormal range $(\geq 0.1 \mathrm{ng} / \mathrm{mL})$. Treatments followed current guidelines [20].

\section{Echocardiographic measurements}

We performed the echocardiographic examination within $24 \mathrm{~h}$ from the ED admission by a standardized protocol, following recommendations of the American and European Societies of Echocardiography.

We previously described the methodology of the acquisition of images $[23,24]$ and the modality of assessment of the left (LV) and right ventricular (RV) systolic function [25-27], as well as measurements of reproducibility [21]. LV systolic dysfunction was defined as LV GLS $\geq-14 \%$, based on the value with a $91 \%$ sensitivity and $75 \%$ specificity for 28-day mortality rate obtained by the ReceiverOperating Characteristics (ROC) curves analysis [28].

Abnormal RV systolic function was defined as TAPSE $<16 \mathrm{~mm}$ [25]. Physicians fully trained in echocardiography (FI, VP) analyzed the images. SIMD was defined as LV GLS $\geq-14 \%$ and/or TAPSE $<16 \mathrm{~mm}$.

\section{Outcomes}

The end-points were day-7 and day-28 all-cause mortalities. We traced medical records within the hospitalization. In case of discharge, we followed all patients by phone calls and chart reviews after hospital discharge, irrespective of re-hospitalizations. No patient was lost at follow-up.

\section{Statistical analysis}

We conducted statistical analyses using IBM SPSS software package (version 25). Data in table are mean values \pm 1 standard deviation of the mean or counts and percent. For continuous variables, the null hypothesis was tested using the Analysis of Variance (ANOVA) test with post hoc analysis. Fisher exact test was used to compare counts in cross tables and the Bonferroni correction was applied for comparisons between three groups. Discrimination ability was tested by ROC curve analysis and the calculation of the Area under the Curve (AUC). Model discrimination was considered suboptimal for areas under the curve $<0.80$. Survival analysis was performed by the Kaplan-Meier analysis to test the association of single variables with day- 7 and day- 28 mortality rates. The variables, which showed a significant 
association with an increased mortality, were analyzed with the multivariate Cox regression method. Two-tailed $p<0.05$ was used to reject the null hypotheses.

\section{Results}

The study cohort comprised 424 patients. From the entire cohort, 19 patients met exclusion criteria, 51 subjects had incomplete echocardiographic assessment of LV and RV function and 29 had known pre-existing LV and/or RV systolic dysfunction. Compared to patients included, those excluded showed a similar SOFA score $(6.4 \pm 2.2$ versus $5.5 \pm 4.9$, respectively, $p>0.1$ ), a comparable proportion of patients with septic shock ( $41 \%$ vs $38 \%$ ), an analogous proportion of all-cause death at day-7 (18\% vs $16 \%)$ and day-28 (31 vs $30 \%$, all $p=$ NS) end-points. Therefore, the study population included 325 patients.

We compared patients included based on 2001 criteria $(n=149)$ and Sepsis-3 $(n=176)$. T0 SOFA (4, [2-6] in both groups), T1 SOFA (4, [2-6] in both groups) as well as day- 7 and day- 28 mortality rates (respectively, $17 \%$ vs $11 \%$ and $33 \%$ vs $25 \%$, all $p=\mathrm{NS}$ ) were similar regardless of the selection criteria and only 2 patients included in the first period had a T0 SOFA $<2$. The study population included 142 patients with normal Troponin level at T0 and T1 (G1 group), 69 patients with abnormal Troponin level either at T0 or T1 (G2 group, 11 patients with abnormal Troponin at T0 and 63 at T1), and 114 patients with Troponin levels increased both at T0 and T1 (G3 group). In Fig. 1, we showed the distribution of $\mathrm{T} 0$ and $\mathrm{T} 1$ Troponin values in the study population.

As reported in Table 1, no significant differences were found in terms of demographic data as well as previous medical conditions among the three groups. Sources of infection were comparable among the three subgroups. SOFA score was lower in G1 than in the other groups. Systolic (SAP) and mean blood pressure (MAP) did not show significant differences between subgroups and a similar proportion of patients in each subgroup had a systolic pressure $>140 \mathrm{mmHg}$ (T0: $13 \%$ in $\mathrm{G} 1,12 \%$ in $\mathrm{G} 2$ and $16 \%$ in $\mathrm{G} 3$; $\mathrm{T} 1: 17 \%$ in $\mathrm{G} 1,20 \%$ in $\mathrm{G} 2$ and $22 \%$ in G3, all $p=\mathrm{NS}$ ). Patients in the 3 different subgroups received a similar amount of fluids as initial bolus and infusion rate. Only at T0, a lower percentage of patients in G1 received the treatment with high-dose vasopressors, compared to the other subgroups. Day-7 and day-28 mortality rates were higher with sustained Troponin increasing (G3) compared to no-Troponin-raise (G1) subgroup, but the difference was significant only for day-28 mortality rate.

$\mathrm{LV}$ and RV parameters of systolic and diastolic function (Table 2) were worse among G3 patients compared to those in G1 and G2 subgroups. LV GLS had a mean value > $-14 \%$ in all subgroups and differed significantly among them.
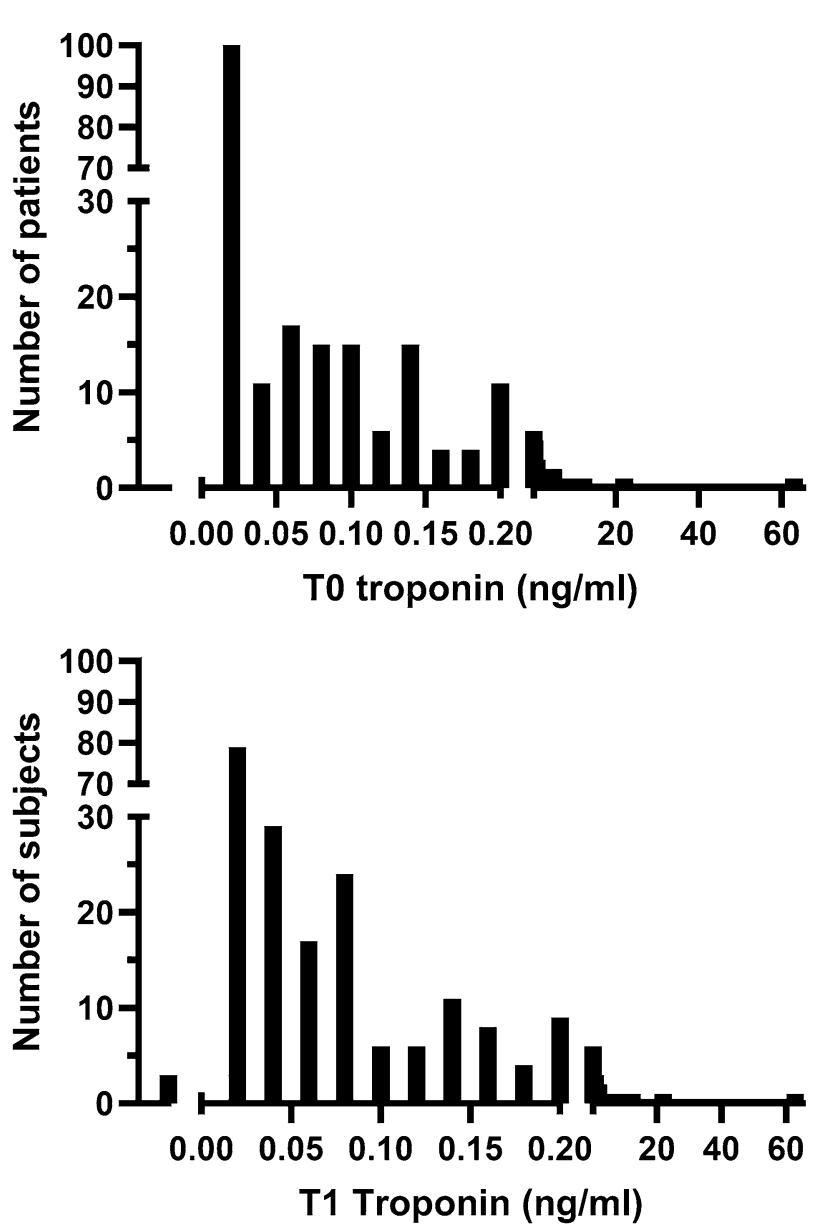

Fig. 1 Distribution of T0 and T1 Troponin values in the study population

Amino-terminal pro-brain natriuretic peptide (NTproBNP) was significantly higher in patients with abnormal levels of Troponin compared to those with normal levels.

In the whole study population, SIMD was detected in 225 patients, 128 with isolated LV systolic dysfunction, 28 with RV systolic dysfunction, and 69 with a biventricular dysfunction. SIMD was more prevalent among G3 patients $(n=96,82 \%)$, compared to $\mathrm{G} 1(n=85,58 \%, p<0.001$ vs G3) and G2 ( $n=44,62 \%, p=0.012$ vs G3). Based on the ROC curves analysis, Troponin showed a just fair but significant discrimination accuracy for SIMD (T0 Troponin: AUC $0.66,95 \%$ CI $0.58-0.73$; T1 Troponin AUC $0.66,95 \%$ CI $0.60-0.72$, all $p<0.001$, Fig. 1 in Supplementary material).

The discrimination of day- 7 and day- 28 mortality rates was non-significant for T0 Troponin (day-7 mortality: AUC $0.59,95 \%$ CI $0.49-0.70, p=0.07$; day-28 mortality: AUC $0.57,95 \%$ CI $0.50-0.65, p=0.06$ ). It was suboptimal and comparable both for T1 Troponin level (day-7 mortality: AUC $0.64,95 \%$ CI $0.54-0.74, p=0.005$; day-28 mortality: T1 Troponin AUC 0.62, 95\% CI 0.55-0.69, $p=0.002$, 
Table 1 Clinical characteristics in the whole population and based on the presence of normal or abnormal $\mathrm{T} 0$ and $\mathrm{T} 1$ Troponin levels

\begin{tabular}{|c|c|c|c|c|}
\hline ] & $\begin{array}{l}\text { All } \\
(n=325)\end{array}$ & $\begin{array}{l}\text { G1 } \\
(n=142)\end{array}$ & $\begin{array}{l}\mathrm{G} 2 \\
(n=69)\end{array}$ & $\begin{array}{l}\text { G3 } \\
(n=114)\end{array}$ \\
\hline Age (years) & $73 \pm 13$ & $70 \pm 14 * \circ$ & $76 \pm 12$ & $76 \pm 11$ \\
\hline Male gender (\%) & $181(56 \%)$ & $82(58 \%)$ & $35(51 \%)$ & $64(56 \%)$ \\
\hline \multicolumn{5}{|l|}{$P M C$} \\
\hline Arterial hypertension (\%) & $174(54 \%)$ & $75(53 \%)$ & $31(45 \%)$ & $68(60 \%)$ \\
\hline Diabetes (\%) & $85(26 \%)$ & $40(28 \%)$ & $14(20 \%)$ & $31(27 \%)$ \\
\hline CAD & $43(13 \%)$ & $18(13 \%)$ & $9(13 \%)$ & $16(14 \%)$ \\
\hline COPD $(\%)$ & $56(17 \%)$ & $30(21 \%)$ & $12(17 \%)$ & $14(12 \%)$ \\
\hline CKD (\%) & $62(19 \%)$ & $23(16 \%)$ & $15(22 \%)$ & $24(21 \%)$ \\
\hline Liver disease (\%) & $18(6 \%)$ & $8(6 \%)$ & $5(7 \%)$ & $5(4 \%)$ \\
\hline Neoplasia (\%) & $97(30 \%)$ & $44(31 \%)$ & $24(35 \%)$ & $29(25 \%)$ \\
\hline \multicolumn{5}{|l|}{ Infection source } \\
\hline Pulmonary (\%) & $159(49 \%)$ & $71(50 \%)$ & $35(51 \%)$ & $53(46 \%)$ \\
\hline Urinary tract $(\%)$ & $62(19 \%)$ & $25(18 \%)$ & $14(20 \%)$ & $23(20 \%)$ \\
\hline Abdominal (\%) & $38(12 \%)$ & $15(11 \%)$ & $5(7 \%)$ & $18(16 \%)$ \\
\hline Soft tissues (\%) & $15(5 \%)$ & $8(6 \%)$ & $2(3 \%)$ & $5(4 \%)$ \\
\hline Devices (\%) & $11(3 \%)$ & $9(6 \%)$ & $2(3 \%)$ & 0 \\
\hline Cardiac (\%) & $4(1 \%)$ & $1(0.1 \%)$ & $1(0.1 \%)$ & $2(0.1 \%)$ \\
\hline Unknown (\%) & $20(6 \%)$ & $6(4 \%)$ & $6(9 \%)$ & $8(7 \%)$ \\
\hline \multicolumn{5}{|l|}{ Sepsis severity } \\
\hline T0 SAP $(\mathrm{mmHg})$ & $109 \pm 25$ & $108 \pm 24$ & $107 \pm 28$ & $112 \pm 25$ \\
\hline T1 SAP $(\mathrm{mmHg})$ & $118 \pm 22$ & $117 \pm 21$ & $116 \pm 22$ & $119 \pm 23$ \\
\hline T0 MAP (mmHg) & $77 \pm 17$ & $77 \pm 17$ & $76 \pm 19$ & $77 \pm 17$ \\
\hline T1 MAP (mmHg) & $78 \pm 14$ & $78 \pm 14$ & $77 \pm 14$ & $78 \pm 15$ \\
\hline T0 SOFA & $6[4-8]$ & $6[3-7]^{* \circ}$ & 7 [5-9] & $6[4-9]$ \\
\hline T1 SOFA & $6[4-8]$ & $5[3-7]^{*}$ & $6[4.8-9]$ & $6[4 \pm 8]$ \\
\hline Septic shock (\%) & $125(38 \%)$ & $44(31 \%)$ & $32(46 \%)$ & $49(43 \%)$ \\
\hline Day-7 mortality (\%) & $43(13 \%)$ & $13(9 \%)$ & $10(14 \%)$ & $20(17 \%)$ \\
\hline Day-28 mortality (\%) & $90(28 \%)$ & $29(20 \%)^{\circ}$ & $22(32 \%)$ & $39(34 \%)$ \\
\hline \multicolumn{5}{|l|}{ Treatment } \\
\hline Fluid bolus (ml) & $1336 \pm 1305$ & $1381 \pm 1013$ & $1302 \pm 1237$ & $1279 \pm 950$ \\
\hline Fluid infusion $(\mathrm{ml} / \mathrm{h})$ & $105 \pm 54$ & $112 \pm 60 *$ & $92 \pm 44$ & $104 \pm 51$ \\
\hline T0 High-dose NA (\%) & $77(24 \%)$ & $22(15 \%) * \circ$ & $21(30 \%)$ & $34(30 \%)$ \\
\hline T1 High-dose NA (\%) & $62(19 \%)$ & $21(15 \%)$ & $17(25 \%)$ & $24(21 \%)$ \\
\hline
\end{tabular}

$P M C$ previous medical conditions, $C O P D$ chronic obstructive pulmonary disease, $C K D$ chronic kidney disease, $C A D$ coronary artery disease, $M A P$ mean arterial pressure, $N A$ noradrenaline ${ }^{*} p<0.05$ between $\mathrm{G} 1$ and $\mathrm{G} 2{ }^{\circ} p<0.05$ between $\mathrm{G} 1$ and $\mathrm{G} 3 ; \S p<0.05$ between $\mathrm{G} 2$ and G3
Fig. 2) as well as for SIMD (day-7 mortality: AUC 0.61, 95\% CI $0.53-0.69, p=0.014$; day-28 mortality AUC $0.61,95 \%$ CI $0.55-0.68, p=0.002$, Fig. 2 in Supplementary material).

According to the Kaplan-Meier analysis, a Troponin level $\geq 0.1 \mathrm{ng} / \mathrm{L}$ (day-7 mortality: 84 vs $91 \%, p=0.052$; day- 28 mortality: 68 vs $78 \%, p=0.042$ ), as well as an age $\geq 65$ years (day-7 mortality: 84 vs $94 \%, p=0.023$; day-28 mortality: 68 vs $85 \%, p=0.007$ ), a SOFA score $\geq 5$ (day-7 mortality: 82 vs $95 \%, p=0.001$; day-28 mortality: 67 vs $81 \%, p=0.007$ ) and the presence of SIMD (day-7 mortality: 82 vs $95 \%, p=0.002$; day-28 mortality: 65 vs $87 \%, p<0.001$ ), were significantly associated with an increased short- and medium-term mortality rate (Fig. 2). When we introduced all these variables in a multivariable Cox survival analysis, the Troponin level did not retain an independent prognostic value (Table 3) while the presence of SIMD showed an independent relationship both with short- and medium-term mortality.

\section{Discussion}

In a population of septic patients, not mechanically ventilated, sustained increment of Troponin level in the first $24 \mathrm{~h}$ was fairly associated with the presence of SIMD defined 
Table 2 Echocardiographic parameters and biomarkers in patients with normal or abnormal Troponin

\begin{tabular}{|c|c|c|c|c|}
\hline & $\begin{array}{l}\text { All patients } \\
(n=325)\end{array}$ & $\begin{array}{l}\text { G1 } \\
(n=142)\end{array}$ & $\begin{array}{l}\text { G2 } \\
(n=69)\end{array}$ & $\begin{array}{l}\mathrm{G} 3 \\
(N=114)\end{array}$ \\
\hline \multicolumn{5}{|l|}{ Echocardiographic parameters } \\
\hline $\operatorname{EDVI}\left(\mathrm{ml} / \mathrm{m}^{2}\right)$ & $41 \pm 16$ & $41 \pm 15$ & $41 \pm 19$ & $42 \pm 170$ \\
\hline LV EF (\%) & $53 \pm 14$ & $56 \pm 12^{\circ}$ & $56 \pm 15 \S$ & $49 \pm 16$ \\
\hline LV GLS (\%) & $-13.0 \pm 3.5$ & $-14.0 \pm 3.5^{\circ}$ & $-13.4 \pm 3.3 \S$ & $-11.6 \pm 3.4$ \\
\hline RV D1 & $3.5 \pm 0.7$ & $3.4 \pm 0.6$ & $3.5 \pm 0.8$ & $3.6 \pm 0.7$ \\
\hline RV D3 & $7.6 \pm 1.2$ & $7.6 \pm 1.1$ & $7.3 \pm 1.3$ & $7.7 \pm 1.1$ \\
\hline TAPSE & $1.8 \pm 0.5$ & $1.9 \pm 0.5^{\circ}$ & $1.8 \pm 0.5$ & $1.8 \pm 0.5$ \\
\hline MV E/A & $1.02 \pm 0.49$ & $1.00 \pm 0.49$ & $0.95 \pm 0.38$ & $1.10 \pm 0.56$ \\
\hline TDI S wave (cm/s) & $7.9 \pm 2.4$ & $8.4 \pm 2.6^{\circ}$ & $8.0 \pm 2.2$ & $7.4 \pm 2.2$ \\
\hline TDI E wave (cm/s) & $8.4 \pm 2.8$ & $9.1 \pm 2.7^{\circ}$ & $8.8 \pm 2.8 \S$ & $7.4 \pm 2.6$ \\
\hline $\mathrm{E} / \mathrm{E}$ ' ratio & $9.5 \pm 5.8$ & $7.9 \pm 3.4^{\circ}$ & $9.2 \pm 5.8$ & $11.7 \pm 7.3$ \\
\hline TR peak gradient $(\mathrm{mmHg})$ & $29 \pm 9$ & $28 \pm 9$ & $28 \pm 9$ & $30 \pm 10$ \\
\hline VCI max diameter (mm) & $1.8 \pm 0.5$ & $1.9 \pm 0.5$ & $1.7 \pm 0.6$ & $1.9 \pm 0.6$ \\
\hline VCI collapsibility index (\%) & $26 \pm 24$ & $24 \pm 23$ & $27 \pm 25$ & $26 \pm 25$ \\
\hline \multicolumn{5}{|l|}{ Biomarkers } \\
\hline T0 NTproBNP (ng/L) & $\begin{array}{l}5810 \\
{[1647-16629]}\end{array}$ & $\begin{array}{l}3417^{\circ} \\
{[1221-8000]}\end{array}$ & $\begin{array}{l}5051 \S \\
{[1107-17336]}\end{array}$ & $\begin{array}{l}11,906 \\
{[4053-27879]}\end{array}$ \\
\hline T1 NTproBNP (ng/L) & $\begin{array}{l}6394 \\
{[2212-17115]}\end{array}$ & $\begin{array}{l}2895 * \circ \\
{[1601-7913]}\end{array}$ & $\begin{array}{l}6581 \S \\
{[3010-19035]}\end{array}$ & $\begin{array}{l}14,707 \\
{[6245-28469]}\end{array}$ \\
\hline T0 Lactate (meq/L) & $3.1 \pm 2.7$ & $2.7 \pm 2.0^{\circ}$ & $3.2 \pm 2.8$ & $3.6 \pm 3.0$ \\
\hline T1 Lactate (meq/L) & $2.0 \pm 2.3$ & $1.7 \pm 2.2$ & $2.0 \pm 1.5$ & $2.5 \pm 2.8$ \\
\hline T0 Troponin (ng/mL) & $\begin{array}{l}0.07 \\
{[0.02-0.29]}\end{array}$ & $\begin{array}{l}0.02 * 0 \\
{[0.02-0.04]}\end{array}$ & $\begin{array}{l}0.07 \S \\
{[0.03-0.14]}\end{array}$ & $\begin{array}{l}0.39 \\
{[0.20-1.28]}\end{array}$ \\
\hline T1 Troponin (ng/mL) & $\begin{array}{l}0.10 \\
{[0.02-0.43]}\end{array}$ & $\begin{array}{l}0.02 * \circ \\
{[0.02-0.04]^{\circ}}\end{array}$ & $\begin{array}{l}0.14 \S \\
{[0.10-0.43]}\end{array}$ & $\begin{array}{l}0.52 \\
{[0.21-1.63]}\end{array}$ \\
\hline
\end{tabular}

All echocardiographic parameters and lactates are reported as mean \pm SD; Troponin and NTproBNP are reported as median and interquartile range

${ }^{*} p<0.05$ between G1 and G2; ${ }^{\circ} p<0.05$ between G1 and G3 mean values; $\$ p<0.05$ between G2 and G3 mean values

$E D V I$ end-diastolic volume index, $L V E F$ left ventricular ejection fraction, $L V G L S$ LV Global Longitudinal Strain, $R V D 1$ right ventricular basal diameter, $R V D 3$ right ventricular longitudinal diameter, TAPSE Tricuspidal Annular Plane Systolic Motion, TDI tissue Doppler imaging, TR tricuspidal regurgitation, VCI inferior vena cava by echocardiographic parameters. In a multivariable model, SIMD predicted independently the mortality in the short and medium term without a contribution of Troponin levels.

We defined LV systolic dysfunction by mean of GLS, a less load-dependent parameter compared to LV EF. Parameters of preload and afterload, including the amount of administered fluids, the E/e' ratio, the collapsibility index of the inferior vena cava, as well as arterial pressure and the proportion of patients treated with high-dose noradrenaline, were similar in patients with normal or abnormal Troponin, while GLS was significantly more compromised in patients with increased levels of the biomarker. We already demonstrated that a reduced LV systolic function defined by GLS was associated with an adverse short- and medium-term outcome. RV systolic function was defined as reduced TAPSE, which already showed a good prognostic value. Therefore, we did not need to adopt different measures, not available at the bedside and difficult to use in an Emergency Medicine setting. Troponin level may be useful to predict prognosis among septic patients in the absence of echocardiography to define SIMD, but the latter seems to have a better association with an unfavorable prognosis, independent to Troponin levels. We are aware that the prognostic discrimination ability was suboptimal for both parameters. During sepsis, the cardiovascular performance plays a pivotal but not unique prognostic role. Several different factors, including previous and concomitant medical conditions, age and the source of the infection heavily influence the possibility of the recovery. SIMD showed a statistically significant correlation to prognosis, so that it might characterize a group of patients, but not describe precisely the prognosis in single patients.

The increase of Troponin levels among critically ill patients is known [4], in particular among septic patients [29]. Nevertheless, we demonstrated that a significant 
Day-7 mortality
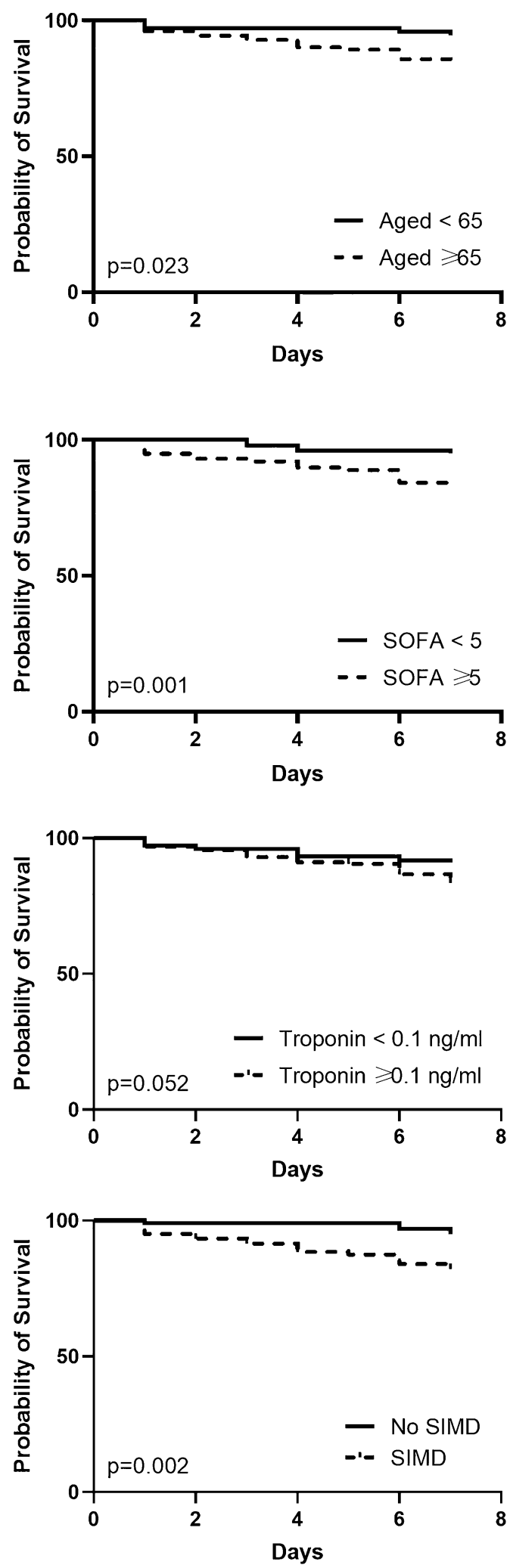

Day-28 mortality
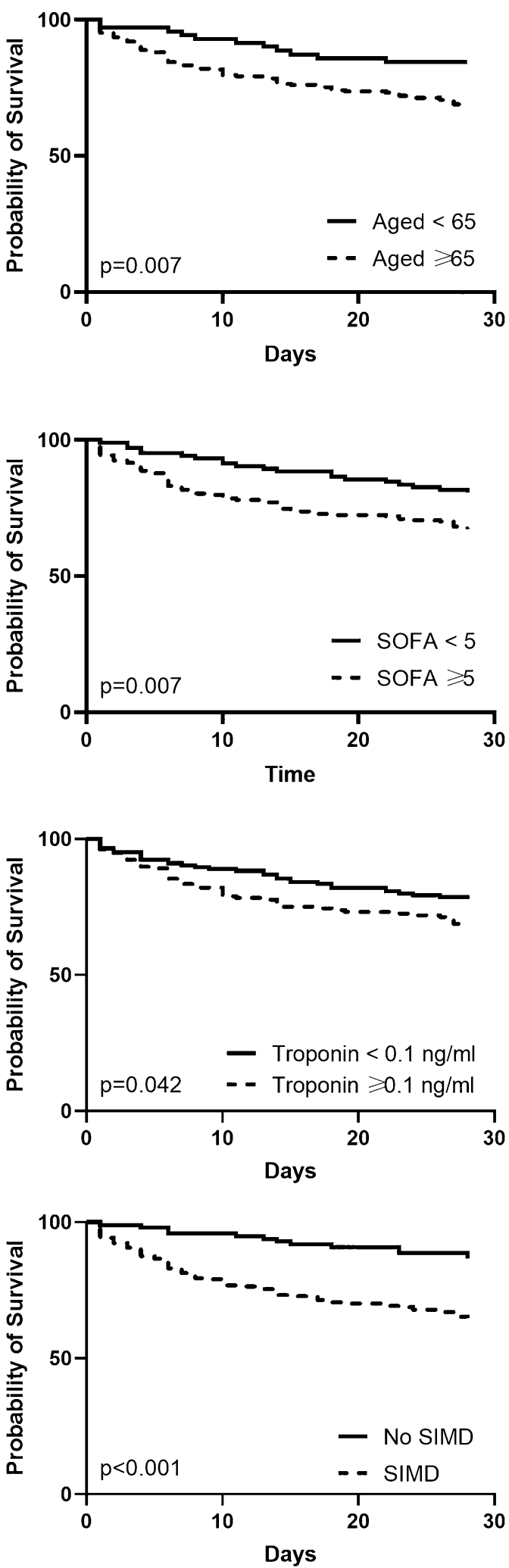

Fig. 2 Prognostic value of abnormal Troponin level and the presence of SIMD evaluated by Kaplan-Meier analysis 
Table 3 Cox regression survival analysis for day-7 and day-28 mortality rates

\begin{tabular}{llll}
\hline & \multicolumn{2}{l}{ Day-7 mortality rate } & \\
\cline { 2 - 4 } & RR & $95 \%$ CI & $p$ \\
\hline Age $\geq 65$ years & - & - & 0.065 \\
T1 SOFA score $\geq 5$ & 3.08 & $1.20-7.89$ & 0.019 \\
T1 Troponin $\geq 0.1 \mathrm{ng} / \mathrm{L}$ & - & - & .274 \\
SIMD & 3.72 & $1.32-10.49$ & 0.013 \\
\hline & Day-28 mortality rate & & \\
\cline { 2 - 4 } & RR & $95 \%$ CI & 0.025 \\
\hline Age $\geq 65$ years & 2.07 & $1.10-3.90$ & \\
T1 SOFA score $\geq 5$ & - & - & 0.062 \\
T1 Troponin $\geq 0.1 \mathrm{ng} / \mathrm{L}$ & - & - & 0.160 \\
SIMD & 3.24 & $1.72-6.11$ & $<0.001$ \\
\hline
\end{tabular}

SOFA Sequential Organ failure Assessment, SIMD sepsis-induced myocardial dysfunction, $R R$ relative risk

portion of patients with SIMD had normal levels of Troponin, while SIMD affected prognosis independently of Troponins. LV/RV dysfunction in sepsis in the absence of a rise in Troponin levels could be equivalent to chronic disease, whereas increase in Troponin levels strongly suggests acute myocardial necrosis independent to pre-existing LV/RV dysfunction. We excluded patients with known pre-existing systolic dysfunction and previous medical conditions showed a similar distribution between subgroups. However, the prevalence of cardiovascular risk factors was considerable and similar between subgroups and we cannot exclude a subclinical impairment of LV longitudinal function independent of the septic event. On the other hand, an underlying CAD, which becomes clinically evident only with a systolic dysfunction, in the absence of an elevation in Troponin levels, is as well an unexpected situation. The fact that Troponin does not predict the prognosis in a multivariable model, including $\mathrm{RV} / \mathrm{LV}$ dysfunction, suggests that the presence of myocardial dysfunction may be stronger than Troponin as global predictor of prognosis including dysfunction as comorbidity as well as acute injury.

Upon ED admission, compared to patients with normal Troponin levels, those with elevated levels showed higher lactate levels as well as an increased SOFA score. These data confirm the relevant role of hemodynamic and cytotoxic mechanisms in promoting sepsis-induced Troponin elevation. At the beginning of the disease, fluids imbalance with consequent tachycardia and hypotension, alongside hypoxemia and reduced oxygen delivery, causes a reduced coronary perfusion pressure and a demand-supply mismatch. In this phase of the disease, elevated lactate levels can be an expression of this imbalance. In the following phases of the resuscitation, elevated filling pressures and increased ventricular wall stress can activate intracellular signaling cascades, with consequent cardiac apoptosis, micronecrosis and increased levels of Troponin. On the other side, the release of cytokines, endotoxins and free radicals exerts a direct toxic effect on myocytes, with altered membrane permeability and apoptosis [30].

The complex interplay between different mechanisms and their different prognostic weight could explain the limited prognostic value of this biomarker $[4,5,8]$. The earliest determination of Troponin lacked a significant prognostic value. A possible interpretation could be the different prognostic weight of an increased level due to a fluid imbalance in the first hours of the resuscitation, compared to an increased secretion due to persistently activated cytotoxic mechanisms, poorly responsive to the treatment [30].

This study has a number of limitations. It is a singlecenter study and, due to the clinical setting where patients were managed, we did not include those who needed invasive ventilation. We cannot exclude that different selection criteria would have changed our results; however, patients like those included in the present study are frequently encountered in the Emergency Medicine setting.

We defined LV systolic dysfunction based on LV GLS, a parameter less load-dependent compared to LV ejection fraction, that cannot be considered derived in common clinical practice in the Emergency Department; however, we found that such a parameter performed bedside may offer useful prognostic indication among patients with sepsis not mechanically ventilated.

We are aware of several limitations of speckle-tracking methodology, especially the need of adequate image quality. However, the analysis could be performed in the majority of our study population and it gave relevant information from a prognostic point of view.

We based our definition of RV systolic dysfunction on TAPSE, which is load dependent: currently, assessment of RV GLS is not available in bedside setting in our clinical 
scenario. A definition of RV dysfunction based on multimodal approach, as recently shown by Vallabhajosyula et al. [31], could add further prognostic information, but it would be less applicable in the Emergency Medicine setting. We focused on relatively fast and widely diffused modality of RV function assessment.

We did not repeat an echocardiographic examination within 24-48 h after the first exam, as our Unit is located within the Emergency Department and patients have to be assigned to their final destination within $48 \mathrm{~h}$. Therefore, in most of them, it was not possible to perform a second examination $24 \mathrm{~h}$ after the first one. We cannot exclude a possible evolution of the LV and RV dysfunction, but the focus of this paper was to find early and reliable parameters, to evaluate the cardiovascular involvement during sepsis and its prognostic value.

\section{Conclusions}

In a population of septic patients not on mechanical ventilation, increased Troponin levels correlated with SIMD diagnosed by echocardiography. However, Troponin did not show an independent association with an increased mortality after adjustment for the presence of SIMD.

Supplementary Information The online version contains supplementary material available at https://doi.org/10.1007/s11739-021-02701-3.

\begin{abstract}
Author contributions F. Innocenti and V. Palmieri gave substantial contributions to the conception and design of the work, drafted and revised the manuscript. F. D’Argenzio, M Cigana, M. Montuori, A. De Paris, E. Capretti and S. Calcagno gave substantial contributions in the acquisition, analysis, or interpretation of data for the work. F. Innocenti, I. Tassinari and V.T. Stefanone drafted the manuscript; V. Palmieri revised it critically for important intellectual content. R. Pini gave the final approval of the version to be published.
\end{abstract}

Funding No funding source to declare.

Availability of data and material The datasets generated during and/or analyzed during the current study are available from the corresponding author on reasonable request.

\section{Declarations}

Conflict of interest The authors declare they have no conflict of interest.

Human and animal rights The study protocol was approved by the "Toscana - Area Vasta-Centro" inter-institutional ethic committee (registration number OSS.13.031) and was conducted in accordance with the Helsinki Declaration of 1964 (revised 2008).

Informed consent All the patients gave informed consent to enter the study.
Consent for publication Patients signed informed consent regarding publishing their data.

\section{References}

1. Yang CW, Li HJ, Thomas L, Ramos M et al (2017) Retrospective cause analysis of troponin I elevation in non-CAD patients Special emphasis on sepsis. Medicine 96:e8027

2. Vasile VC, Chai HS, Abdeldayem D, Afessa B et al (2013) Elevated cardiac troponin $\mathrm{T}$ levels in critically Ill patients with sepsis. Am J Med 126:1114-1121

3. Cheng H, Fan WZ, Wang SC, Liu ZH et al (2015) N-terminal pro-brain natriuretic peptide and cardiac troponin I for the prognostic utility in elderly patients with severe sepsis or septic shock in intensive care unit: A retrospective study. J Crit Care 30(654):e659-e614

4. Masson S, Caironi P, Fanizza C, Carrer S et al (2016) Sequential N-Terminal Pro-B-type natriuretic peptide and high-sensitivity cardiac troponin measurements during albumin replacement in patients with severe sepsis or septic shock. Crit Care Med 44:707-716

5. Vallabhajosyula S, Sakhuja A, Geske JB, Kumar M et al (2017) Role of admission troponin-T and serial troponin- $\mathrm{T}$ testing in predicting outcomes in severe sepsis and septic shock. J Am Heart Assoc 6:e005930

6. Sheyin O, Davies O, Duan W, Perez X (2015) The prognostic significance of troponin elevation in patients with sepsis: a metaanalysis. Heart Lung 44:75-81

7. Wilhelm J, Hettwer S, Schuermann M, Bagger S et al (2014) Elevated troponin in septic patients in the emergency department: frequency, causes, and prognostic implications. Clin Res Cardiol 103:561-567

8. Rosjo H, Varpula M, Hagve TA, Karlsson S et al (2011) Circulating high sensitivity troponin $\mathrm{T}$ in severe sepsis and septic shock: distribution, associated factors, and relation to outcome. Intensive Care Med 37:77-85

9. Scott EC, Ho HC, Yu M, Chapital AD et al (2008) Pre-existing cardiac disease, troponin I elevation and mortality in patients with severe sepsis and septic shock. Anaesth Intensive Care 36:51-59

10. Yucel T, Memis D, Karamanlioglu B, Sut N et al (2008) The prognostic value of atrial and brain natriuretic peptides, troponin I and C-reactive protein in patients with sepsis. Exp Clin Cardiol 13:183-188

11. Brivet FG, Jacobs FM, Colin P, Prat D et al (2006) Cardiac troponin level is not an independent predictor of mortality in septic patients requiring medical intensive care unit admission. Crit Care 10:404

12. Kim JS, Kim M, Kim YJ, Ryoo SM et al (2019) Troponin testing for assessing sepsis-induced myocardial dysfunction in patients with septic shock. J Clin Med 8:239

13. Mehta NJ, Khan IA, Gupta V, Jani K et al (2004) Cardiac troponin I predicts myocardial dysfunction and adverse outcome in septic shock. Int J Cardiol 95:13-17

14. ver Elst KM, Spapen HD, Nguyen DN, Garbar C et al (2000) Cardiac troponins I and T are biological markers of left ventricular dysfunction in septic shock. Clin Chem 46:650-657

15. Landesberg G, Levin PD, Gilon D, Goodman S et al (2015) Myocardial dysfunction in severe sepsis and septic shock: no correlation with inflammatory cytokines in real-life clinical setting. Chest 148:93-102

16. Charpentier J, Luyt CE, Fulla Y, Vinsonneau C et al (2004) Brain natriuretic peptide: A marker of myocardial dysfunction and prognosis during severe sepsis. Crit Care Med 32:660-665 
17. De Geer L, Engvall J, Oscarsson A (2015) Strain echocardiography in septic shock - a comparison with systolic and diastolic function parameters, cardiac biomarkers and outcome. Critical Care 19

18. Levy MM, Fink MP, Marshall JC, Abraham E et al (2003) 2001 SCCM/ESICM/ACCP/ATS/SIS International sepsis definitions conference. Crit Care Med 31:1250-1256

19. Seymour CW, Liu VX, Iwashyna TJ, Brunkhorst FM et al (2016) Assessment of clinical criteria for sepsis: for the third international consensus definitions for sepsis and septic shock (Sepsis-3). JAMA 315:762-774

20. Singer M, Deutschman CS, Seymour CW, Shankar-Hari M et al (2016) The third international consensus definitions for sepsis and septic shock (Sepsis-3). JAMA 315:801-810

21. Palmieri V, Innocenti F, Guzzo A, Guerrini E et al (2015) Left ventricular systolic longitudinal function as predictor of outcome in patients with sepsis. Circ Cardiovasc Imaging 8:e003865

22. Innocenti F, Tozzi C, Donnini C, De VE et al (2017) SOFA score in septic patients: incremental prognostic value over age, comorbidities, and parameters of sepsis severity. Intern Emerg Med 13:405-412

23. Voigt JU, Pedrizzetti G, Lysyansky P, Marwick TH et al (2015) Definitions for a common standard for 2D speckle tracking echocardiography: consensus document of the EACVI/ASE/Industry Task Force to standardize deformation imaging. J Am Soc Echocardiogr 28:183-193

24. Innocenti F, Palmieri V, Stefanone VT, Donnini C et al (2020) Epidemiology of right ventricular systolic dysfunction in patients with sepsis and septic shock in the emergency department. Intern Emerg Med 15:1281-1289

25. Rudski LG, Lai WW, Afilalo J, Hua L et al (2010) Guidelines for the echocardiographic assessment of the right heart in adults: a report from the American Society of Echocardiography endorsed by the European Association of Echocardiography, a registered branch of the European Society of Cardiology, and the Canadian Society of Echocardiography. J Am Soc Echocardiogr 23:685-713
26. Nagueh SF, Smiseth OA, Appleton CP, Byrd BF et al (2016) Recommendations for the evaluation of left ventricular diastolic function by echocardiography: an update from the american society of echocardiography and the european association of cardiovascular imaging. Eur Heart J-Card Img 17:1321-1360

27. Lang RM, Badano LP, Mor-Avi V, Afilalo J et al (2015) Recommendations for cardiac chamber quantification by echocardiography in adults: an update from the American Society of Echocardiography and the European Association of Cardiovascular Imaging. J Am Soc Echocardiogr 28(1-39):e14

28. Innocenti F, Palmieri V, Guzzo A, Stefanone VT et al (2018) SOFA score and left ventricular systolic function as predictors of short-term outcome in patients with sepsis. Intern Emerg Med 13:51-58

29. Klouche K, Pommet S, Amigues L, Bargnoux AS et al (2014) Plasma brain natriuretic peptide and troponin levels in severe sepsis and septic shock: relationships with systolic myocardial dysfunction and intensive care unit mortality. J Intensive Care Med 29:229-237

30. Zochios V, Valchanov K (2015) Raised cardiac troponin in intensive care patients with sepsis, in the absence of angiographically documented coronary artery disease: A systematic review. J Intensive Care Soc 16:52-57

31. Vallabhajosyula S, Kumar M, Pandompatam G, Sakhuja A et al (2017) Prognostic impact of isolated right ventricular dysfunction in sepsis and septic shock: an 8-year historical cohort study. Ann Intensive Care 7:94

Publisher's Note Springer Nature remains neutral with regard to jurisdictional claims in published maps and institutional affiliations. 